\title{
SÉQUELLES DE LA COVID 19 DANS LE GOÛT ET L'ODORAT: UNE BRÈVE REVUE DE LA LITTÉRATURE
}

\section{REVUE DE LITTÉRATURE}

CHAVES, leda Bezerra ${ }^{1}$, FECURY, Amanda Alves², OLIVEIRA, Euzébio de ${ }^{3}$, DENDASCK, Carla Viana ${ }^{4}$, DIAS, Claudio Alberto Gellis de Mattos ${ }^{5}$

CHAVES, leda Bezerra. Et al. Séquelles de la COVID 19 dans le goût et l'odorat: une brève revue de la littérature. Revista Científica Multidisciplinar Núcleo do Conhecimento. Année. 06, Ed. 11, vol. 01, p. 150 à 166. Novembre 2021. ISSN : 2448-0959, Lien d'accès:

https://www.nucleodoconhecimento.com.br/sante/sequelles-de-la-covid-19, $\quad$ DOI: 10.32749/nucleodoconhecimento.com.br/sante/sequelles-de-la-covid-19

\section{RÉSUMÉ}

Les virus sont un être vivant qui n'est pas formé par la structure cellulaire. Les virus peuvent être transmis par contact direct avec des personnes infectées ou indirectement à partir de surfaces contaminées. Défini comme une nouvelle variété de coronavirus, le COVID 19 a fait déclarer à l'Organisation mondiale de la santé (OMS) début 2020 une crise de santé publique mondiale. Compte tenu de la haute transmissibilité de ce virus, il était nécessaire d'adopter des mesures pour prévenir la transmission et l'infection. Les symptômes causés par la COVID-19 sont

\footnotetext{
${ }^{1}$ Étudiant du lycée technique intégré en alimentation, Institut d'éducation de base, technique et technologique d'Amapá (IFAP).

${ }^{2}$ Biomédical, $\mathrm{PhD}$ en maladies topiques, professeur et chercheur du cours de médecine du campus de Macapá, Université fédérale d'Amapá (UNIFAP).

${ }^{3}$ Biologiste, PhD en maladies topiques, professeur et chercheur du cours d'éducation physique, Université fédérale du Pará (UFPA).

${ }^{4}$ Théologien, Doctorat en psychanalyse clinique. II travaille depuis 15 ans avec la méthodologie scientifique (méthode de recherche) dans le cadre de l'orientation de production scientifique des étudiants à la maîtrise et au doctorat. Spécialiste en études de marché et en recherche axée sur la santé. Doctorant en Communication et Sémiotique (PUC SP).

${ }^{5}$ Biologiste, Ph.D. en recherche théorique et comportementale, professeur et chercheur du cours de chimie de I'Institut d'éducation fondamentale, technique et technologique de l'Amapá (IFAP) et du Graduate Program in Professional and Technological Education (PROFEPT IFAP).
}

RC: 101283

Disponible en: https://www.nucleodoconhecimento.com.br/sante/sequelles-de-la$\underline{\text { covid-19 }}$ 
habituellement de la fièvre, de la toux sèche, de l'essoufflement (dyspnée), de la fatigue (fatigue), des maux de gorge, des maux de tête et de la diarrhée. D'autres symptômes, dans une moindre mesure, sont l'anosmie (perte de l'odorat), l'hyposmie (diminution de l'odorat) et l'ageusia (perte du sens du goût). L'objectif était de passer en revue les deux dernières années sur les séquelles de la COVID 19 dans le goût et l'odorat. Une brève revue de la littérature a été menée dans des articles scientifiques sur les séquelles du COVID 19 dans le goût et l'odorat, entre 2020 et 2021, en portugais, dans la base de données de recherche Google Scholar. Les femmes et les jeunes sont les plus touchés par les séquelles du goût et de l'odorat causées par la COVID-19. II n'existe pas de traitements spécifiques et scientifiquement prouvés aux différents dysfonctionnements, mais le plus indiqué est la pratique de l'entraînement olfactif. Les études liées aux dysfonctionnements sont limitées, principalement, au Brésil et lorsqu'elles sont analysées comme des séquelles. La continuité de la recherche scientifique est indispensable, car seul ce processus permettra d'élucider les doutes qui subsistent et de générer des traitements appropriés pour les personnes ayant développé la COVID-19.

Mots-clés: Virus, COVID 19, Dysosmie, Dysgueusie.

\section{INTRODUCTION}

Les virus sont un être vivant qui, contrairement aux autres, n'est pas formé par la cellule. II a généralement un acide nucléique (qui peut être de l'ADN ou de l'ARN), recouvert d'une «capsule » protéique. Certains sont encore recouverts de « morceaux » de membrane cellulaire, qui créent une " enveloppe » autour d'elle. Ils sont souvent plus petits qu'une cellule et les parasitent, y pénètrent et utilisent leurs structures pour produire plus de virus (ce sont des parasites intracellulaires obligatoires) (Brandão, 2015; Eux, 2020).

Les virus peuvent être transmis par contact direct avec des personnes infectées ou indirectement à partir de surfaces contaminées. Ils sont transmis d'un individu à un autre par des gouttelettes d'eau sous forme de vapeur libérée dans l'haleine ou 
pendant le processus de toux ou d'éternuement. De plus petites gouttelettes libérées avec de la vapeur d'eau au cours de ces processus sont suspendues dans l'air, propageant le virus sur de plus grandes surfaces et plus longtemps. (Brasil, 2021). Au contact de l'un de ces moyens de transmission, les virus envahissent les cellules muqueuses des voies respiratoires et des digestórias (Stephens et al., 2009; Silva et al., 2020).

Pour être reproducteurs, les virus se lient à la surface de la membrane cellulaire. Après cette liaison, il peut pénétrer dans la cellule ou «injecter " son matériel génétique (ADN ou ARN) dans son cytoplasme. Une fois à l'intérieur des cellules, le matériel génétique du virus est copié plusieurs fois, en utilisant les réactions chimiques qu'il peut faire. De même, d'autres réactions chimiques de la cellule sont utilisées pour fabriquer les "couvertures» de protéines (capsides). Ainsi, de nouveaux virus sont montés à l'intérieur d'une cellule et, pour sortir et envahir de nouvelles cellules, briser la membrane de ceux qui les ont fabriqués (They, 2020; Silva et al., 2020a).

Défini comme une nouvelle variété de coronavirus, le COVID 19 a fait déclarer à l'Organisation mondiale de la santé (OMS) début 2020 une crise de santé publique mondiale (Souza et al., 2020). Ces virus, avec un taux de transmission élevé, sont constitués d'enveloppes de lipides et de protéines (morceau de membrane d'une cellule), et de capsides protéiques, avec un seul ruban d'ARN (acide nucléique), et avec une forme de couronne externe (Silva et al., 2020). Ils font partie de la famille des virus qui génèrent le rhume à des infections plus graves, telles que le syndrome respiratoire du Moyen-Orient (MERS) et le syndrome respiratoire aigu sévère (SRAS) (Brésil, 2021; 2021a).

Compte tenu de la haute transmissibilité de ce virus, il est nécessaire d'adopter des mesures pour prévenir la transmission et l'infection. Parmi certaines de ces actions préventives, nous soulignons la non-permanence dans les endroits fermés et l'isolement des personnes infectées (Stephens et al., 2009). 
Les moyens considérés comme les plus efficaces et d'éviter la contamination (prophylaxie) comprennent: le lavage des mains, la distanciation sociale, l'aération des environnements et leur exposition au soleil, le nettoyage des objets et des surfaces, l'interdiction des agglomérations et l'utilisation de masques (bloqueurs efficaces des gouttelettes respiratoires dispersées, même à des distances réduites avec contact direct ou indirect) (Garcia, 2020). Ces mesures de lutte se sont avérées efficaces, grâce à des analyses et des études scientifiques, pour réduire la contamination et la dissémination virale (Soares et al., 2021).

Les symptômes causés par la COVID-19 en général sont la fièvre, la toux sèche, l'essoufflement (dyspnée), la fatigue (fatigue), les maux de gorge, les maux de tête et la diarrhée (Silva et coll., 2020). D'autres symptômes, dans une moindre mesure, sont l'anosmie (perte de l'odorat), l'hyposmie (diminution de l'odorat) et l'ageusia (perte du sens du goût) (Iser et al., 2020).

Les séquelles peuvent être définies comme un changement dans le fonctionnement des cellules et/ou dans la forme et le fonctionnement des organes causant un défaut permanent ou non chez l'individu (Filho, 2011; Vana et Schiozer, 2013).

Parmi les diverses séquelles trouvées chez les personnes atteintes de COVID19 figurent celles de nature psychologique et biologique, telles que des changements dans les systèmes respiratoire, cardiovasculaire, cutanés possibles et neurologiques (dépression et anxiété) (Estrela et al., 2021).

Un ensemble de recherches scientifiques indique des dommages à long terme (après 6 semaines ou persistant au-delà de 6 semaines à compter de l'apparition des symptômes du coronavirus) dans le système sensoriel, en mettant l'accent sur les séquelles olfactives et gustatives, par exemple la dysosmie (perception déformée des odeurs) et la dysgueusie (perception déformée des goûts alimentaires). II peut également y avoir des troubles du métabolisme du glucose (réactions chimiques), une hyperlipidémie (augmentation de la quantité de graisse dans le sang), un dérèglement du métabolisme des lipides (réactions chimiques), un trouble de stress 
post-traumatique (l'un des types de troubles anxieux) et une capacité pulmonaire affectée, des dommages au foie (foie) et au système rénal (reins), et éventuellement dans le système reproducteur (Sesapr, 2020; Grendene et al., 2021).

Les dysfonctionnements olodorants ont une classification quantitative, dans laquelle il y a anosmie (pas de détection des odeurs), hyposmie (limitation dans la détection des odeurs), hyperosmie (augmentation de la capacité à détecter les odeurs) et dysosmie (perception déformée des odeurs) (Cardoso, 2018). Alors que les dysfonctionnements quantitatifs du goût peuvent être classés comme hypogéusie (détection partielle du goût), hypergéusie (augmentation de la capacité de perception gustative), ageusia (perte totale du goût) et dysgueusie (distorsion du goût) (Barros et al., 2015; Gomes et al., 2020; Machado e Machado, 2020).

La sous-classification qualitative de la dysosmie (altération olfactive) est diverse, parmi les exemples on peut trouver la parosmie (perception déformée et désagréable des odeurs), la fantosmie (perception des odeurs en l'absence de particules odoriférantes) et l'agnosmie (incapacité à classer différentes odeurs) (Cardoso, 2018). La dysgueusie (altération gustative) a une sous-classification qualitative, également diversifiée, entre exemples, la parageusie (perception inadéquate du goût due au stimulus) et la fantogeusie (perception gustative désagréable en l'absence de stimulus) (Machado e Machado, 2020).

Malgré les notes, d'autres études sont nécessaires en ce qui concerne les nombreuses et diverses séquelles dues au covid-19 (Grendene et al., 2021), y compris les personnes atteintes de symptômes réduits et asymptomatiques (Uzun, 2020).

\section{OBJECTIF}

Faites une revue de la littérature des deux dernières années sur les séquelles de la COVID 19 dans le goût et l'odorat. 


\section{MÉTHODE}

Une brève revue de la littérature a été menée dans des articles scientifiques sur les séquelles du COVID 19 dans le goût et l'odorat, entre 2020 et 2021, en portugais, dans la base de données de recherche Google Scholar. Les mots-clés (ou descripteurs) « Gustation et COVID19 » et «Odeur et COVID 19 » ont été utilisés. Au total, 1777 résultats ont été trouvés et 10 articles ont été utilisés pour constituer cette brève revue. Cela a permis de faire l'examen sur la base des productions académiques les plus récentes (Capes, 2012).

Des articles avec un contenu COVID 19 et des séquelles dans le goût et l'odeur ont été utilisés, en portugais, dans la limite de la période de l'examen. Les articles qui ne répondaient pas à ce contenu et/ou à cette période de temps ont été exclus.

\section{RÉSULTATS ET DISCUSSION}

Le tableau 01 indique le nombre d'articles trouvés par décrits et le nombre d'articles utilisés dans les critères méthodologiques. 
Tableau 1 - Nombre d'articles trouvés dans la base de données selon les descripteurs et les articles utilisés

\begin{tabular}{|c|c|c|c|c|}
\hline BASE DE DADOS & DESCRITOR & $\begin{array}{c}\text { NUMERO } \\
\text { ARTIGOS } \\
\text { ENCONTRADOS }\end{array}$ & $\begin{array}{c}\text { NUMERO } \\
\text { ARTIGOS } \\
\text { UTILIZADOS }\end{array}$ & \begin{tabular}{|c|} 
AUTOR E ANO DOS \\
ARTIGOS \\
UTILIZADOS
\end{tabular} \\
\hline \multirow{10}{*}{ Google acadêmico } & \multirow{7}{*}{ Gustação e COVD19 } & \multirow{7}{*}{237} & \multirow{7}{*}{7} & PIMENTEL, 2020 \\
\hline & & & & $\begin{array}{c}\text { GRENDENE et al., } \\
2021\end{array}$ \\
\hline & & & & SANTOS et al., 2020 \\
\hline & & & & COSTA et al., 2020 \\
\hline & & & & SOUZA et al., 2021 \\
\hline & & & & $\begin{array}{c}\text { NOGUEIRA et al., } \\
2021\end{array}$ \\
\hline & & & & NUNES et al., 2020 \\
\hline & \multirow{3}{*}{ Olfato e COVID 19} & \multirow{3}{*}{1540} & \multirow{3}{*}{3} & KOSUGI et al., 2020 \\
\hline & & & & BRITO e SLVA, 2020 \\
\hline & & & & $\begin{array}{c}\text { LOPES e DE ABREU, } \\
2021\end{array}$ \\
\hline
\end{tabular}

RC: 101283

Disponible en: https://www.nucleodoconhecimento.com.br/sante/sequelles-de-lacovid-19 
Le tableau 02 - présente les méthodes utilisées dans chaque article sélectionné pour cette revue, les résultats et les conclusions trouvés.

\begin{tabular}{|c|c|c|c|}
\hline $\begin{array}{c}\text { AUTOR E ANO } \\
\text { DOS ARTIGOS } \\
\text { UTILIZADOS }\end{array}$ & $\begin{array}{l}\text { TIPO DE } \\
\text { MÉTODO }\end{array}$ & RESULTADOS & CONCLUSÃO \\
\hline PIMENTEL, 2020 & $\begin{array}{l}\text { Pesquisa } \\
\text { qualitativa } \\
\text { descritiva, } \\
\text { revisão } \\
\text { bibliográfica da } \\
\text { literatura }\end{array}$ & \begin{tabular}{|} 
Estudos dassificaram as disfunções como: \\
anosmia/hiposmia ou ageusia e \\
hipogeusia/disgeusia. Pacientes tiveram os sintomas \\
disfuncionais tanto no olfato quanto na gustação. \\
Não são considerados danos permanentes.
\end{tabular} & $\begin{array}{l}\text { Identificação hiposmia, anosmia, fantosmia e } \\
\text { parosmia, com preval ência entre } 22,7 \% \text { a } 88,8 \% \text {, } \\
\text { associadas ou não hipogeu sia e ag eusia Futuros } \\
\text { estudos epi demi ológicos devem esclarecer como } \\
\text { esses sintomas são causados. }\end{array}$ \\
\hline $\begin{array}{l}\text { SANTOS et al }= \\
2020\end{array}$ & $\begin{array}{c}\text { Revisão } \\
\text { Integrativa da } \\
\text { literatura }\end{array}$ & $\begin{array}{c}\text { Preval ência das disfunções sensoriais provocadas } \\
\text { pela COVID-19. Anosmia e disgeusia como } \\
\text { sintomas neurológicos mais frequentes. Pacientes } \\
\text { que tiveram quadro leve a moderado de COVID-19. } \\
\text { Aumento da idade dos pacientes e quadros mais } \\
\text { severos da doença representam menos al terações. }\end{array}$ & $\begin{array}{l}\text { Sintese de informações de vários pai ses, } \\
\text { caracterizando as disfunções olfativas e gustativas } \\
\text { como sintomas iniciais. Repercu ssões após alta dos } \\
\text { pacientes. Necessários estudos sobre as } \\
\text { probl emáticas e investigações mais detalhadas. Os } \\
\text { distúrbios poderão ser estudados em um periodo de } \\
\text { tempo maior. }\end{array}$ \\
\hline $\begin{array}{l}\text { NOGUEIRA et al ., } \\
2021\end{array}$ & $\begin{array}{l}\text { Revisão } \\
\text { descritiva }\end{array}$ & $\begin{array}{c}\text { A infecção nos neurônios causa sintomas } \\
\text { neurológicos como anosmia. Não há explicação } \\
\text { exata acerca da ocorrência dos distúrbios olfatórios. } \\
\text { Danos no sistema nervoso central e epitélio nasal, } \\
\text { cau sam a disosmia e a disgeusia. }\end{array}$ & $\begin{array}{l}\text { Os probl emas olfativos e gustativo ocorrem } \\
\text { previamente em diferentes intensi dades. Não há } \\
\text { evidências cientificas para o tratamento desses } \\
\text { distúrbios. É importante a realização de estudos } \\
\text { acerca do assunto a fim de que se melhore os } \\
\text { procedimentos no tratamento nos casos, } \\
\text { principalmente, nos casos com sequelas de longo } \\
\text { prazo. }\end{array}$ \\
\hline $\begin{array}{c}\text { COSTA et al., } \\
2020\end{array}$ & $\begin{array}{l}\text { Revisão } \\
\text { Sistemática }\end{array}$ & $\begin{array}{l}\text { Sintomas neurológicos que ocorrem no sistema } \\
\text { nervoso periférico causam as disfunções olfativa e } \\
\text { gustativa. Pacientes com quadro leve a moderado } \\
\text { apresentaram hipogeusia e hiposmia, não há } \\
\text { registros do inicio, duração e resolução dos } \\
\text { sintomas. Os pacientes com perda de olfato tiveram } \\
\text { menor taxa de internação, compar ados com os } \\
\text { pacientes sem perda de olfato. }\end{array}$ & $\begin{array}{l}\text { Os distúrbios olfativos e/ou gustativos podem } \\
\text { ocorrer em intensidades variáveis, com inicio junto } \\
\text { com os sintomas prévios da infecção. Não há } \\
\text { evidências cientificas acerca do tratamento destes } \\
\text { distúrbios. }\end{array}$ \\
\hline
\end{tabular}

RC: 101283

Disponible en: https://www.nucleodoconhecimento.com.br/sante/sequelles-de-lacovid-19 


\begin{tabular}{|c|c|c|c|}
\hline $\begin{array}{c}\text { BRITO e SILA, } \\
2020\end{array}$ & $\begin{array}{l}\text { Revisão da } \\
\text { literatura }\end{array}$ & $\begin{array}{l}\text { Associação de manifestações neurológicas e a } \\
\text { COVID-19. Entre elas os distúrbios do olfato e } \\
\text { paladar, como a hiposmia, a anosmia, a disg eusia, } \\
\text { hipogeusia e ageusia. Os mecanismos exatos da } \\
\text { neuroinvasão causada pela COVID-19 ainda são } \\
\text { desconhecidos. }\end{array}$ & $\begin{array}{l}\text { Ocorre uma inci dência maior de danos no sistema } \\
\text { nervoso em quadros agudos da virose. Entretanto a } \\
\text { classificação dos casos agudos e crônicos ainda hoje } \\
\text { não é consenso. São necessários mais estu dos sobre } \\
\text { as sequelas a longo prazo. }\end{array}$ \\
\hline $\begin{array}{c}\text { SOUZA et al., } \\
2021\end{array}$ & Relato de casos & $\begin{array}{l}\text { Anosmia nos pacientes é uma manifestação } \\
\text { sintomal ógica recorrente. Fundamental a descrição } \\
\text { de relatos de casos a fim de esclarecer as } \\
\text { ocorrências de sequelas gustativas olfativas com } \\
\text { objetivo de realializar o manejo da recuperação. }\end{array}$ & $\begin{array}{l}\text { As disfunções devem ser estudadas com maior rigor } \\
\text { e por mai or periodo de tempo. A COVD-19 afeta } \\
\text { as células do epitélio olf ativo, possivelmente } \\
\text { alterando a neurotransmissão. }\end{array}$ \\
\hline $\begin{array}{l}\text { LOPES e DE } \\
\text { ABREU } 2021\end{array}$ & $\begin{array}{c}\text { Metodologia para } \\
\text { tratamento }\end{array}$ & $\begin{array}{l}\text { Possiveis danos neurológicos em nivel celular } \\
\text { decorrentes da COVID-19. Pacientes recuperados } \\
\text { ainda relatam alterações, que podem ser } \\
\text { irreversiveis, nos senti dos olf ativo e gustativo. }\end{array}$ & $\begin{array}{l}\text { As sequelas causadas pela doença podem ser de } \\
\text { cunho neurológicos, em nivel celular ou não. Isso } \\
\text { pode causar transtornos, sindromes e outras } \\
\text { variáveis futuramente. }\end{array}$ \\
\hline $\begin{array}{l}\text { NUNES et al., } \\
2020\end{array}$ & $\begin{array}{l}\text { Revisão } \\
\text { Integrativa }\end{array}$ & $\begin{array}{l}\text { Disfunções olfativas com preval ência entre } 30 \text { a } \\
88 \% \text { dos pacientes com COVID-19 englobados nos } \\
\text { diferentes estudos. Relados por pacientes com } \\
\text { quadros leves e moderados da doença. Os } \\
\text { transtornos como anosmia, hiposmiahipogeusia, e } \\
\text { disgeusia são sintomas frequentes nos pacientes } \\
\text { com COVID-19. }\end{array}$ & $\begin{array}{l}\text { Oenvolvimento do virus com o sistema nervoso é, } \\
\text { até então, inconclusivo. Entender os sintomas } \\
\text { neuroló gicos, principalmente em quadros mais } \\
\text { graves, auxilia nas intervenções e nos prognósticos } \\
\text { médicos. }\end{array}$ \\
\hline $\begin{array}{c}\text { GRENDENE et } \\
\text { al., } 2021\end{array}$ & $\begin{array}{c}\text { Revisão } \\
\text { bibliográfica }\end{array}$ & $\begin{array}{c}\text { Aponta que existem poucos estudos revisados sobre } \\
\text { a ocorrência ou prevalência das sequelas de longo } \\
\text { prazo da COVID-19. Há algumas evidências de que } \\
\text { disfunções como a disosmia e đisgeusia são } \\
\text { sequelas de longo prazo associadas à COVID-19. A } \\
\text { mai oria não trabalha com periodos de } \\
\text { acompanhamento indicativos de sequelas de longo } \\
\text { prazo. }\end{array}$ & $\begin{array}{l}\text { Não se sabe muito sobre estratégias para tratamento } \\
\text { para este virus. Pesquisas clinicas estão sendo } \\
\text { realizadas para descobrir novos tratamentos. } \\
\text { Prevenção ainda é a melhor forma de lidar. }\end{array}$ \\
\hline $\begin{array}{l}\text { KOSUGI et al ., } \\
2020\end{array}$ & $\begin{array}{l}\text { Quali quantitativa } \\
\text { por questionário }\end{array}$ & $\begin{array}{l}\text { Induidos } 253 \text { pacientes, de todas as regiões } \\
\text { br asileiras, uma maioria apresentou anosmia e } 13 \text {, } \\
2 \% \text { não tiveram recuperação da perda olfativa. } \\
\text { Alteração no paladar não está induida na pesquisa. }\end{array}$ & $\begin{array}{l}\text { Anosmia apresenta menor taxa de recu peração e } \\
\text { duração mai or em pacientes por COVD-19 } \\
\text { positivos do que nos negativos. Hiposmia teve } \\
\text { maior taxa de recuper ação do que a anosmia. }\end{array}$ \\
\hline
\end{tabular}

Grâce à des études Pimentel (2020) basées sur 15 publications décrivant des rapports cliniques en Europe début 2020, des cas d'hyposmie, d'anosmie, de fantosmie et de parosmie ont été identifiés, avec une prévalence comprise entre $22,7 \%$ et $88,8 \%$, associée ou non à une hypogésie et à une agéousie.

L'amplitude des taux de symptômes sensoriels / séquelles est due à la différence entre les études, ainsi qu'aux instruments utilisés par ces recherches scientifiques variées, et même aux différences génétiques dans la population (Pimentel, 2020). Les différences entre les populations montrent que les taux d'apparition d'anomalies du goût olfactif sont plus élevés, par exemple, dans les populations d'Europe, d'Afrique du Nord, d'Asie du Sud et d'Asie occidentale, par rapport aux taux les plus bas présentés par les patients d'Asie de l'Est (Santos et al., 2020). Les femmes et

RC: 101283

Disponible en: https://www.nucleodoconhecimento.com.br/sante/sequelles-de-la$\underline{\text { covid-19 }}$ 
les jeunes semblent être les plus touchés par les troubles olfactifs et gustatifs (Costa et al., 2020; Nascimento, 2020). Cette différence se produit probablement parce qu'il existe une différence dans la réaction de défense physiologique entre les groupes d'âge et les genres de l'espèce humaine (Cardoso et al., 2020).

L'étude de Santos et al. (2020) souligne que les dysfonctionnements olfactifs et gustatifs sont des symptômes initiaux, et qu'ils ont une plus grande répercussion après la sortie des patients.

Le traitement hospitalier des patients se termine avant l'apparition de séquelles, probablement parce qu'il existe une carence historique de postes vacants et de professionnels de la santé au Brésil. L'augmentation du flux hospitalier de patients pendant la pandémie semble avoir aggravé la situation, empêchant un suivi spécifique et approfondi, car les soins étaient déjà précaires (Santo et al., 2021).

Dans la revue intégrative de Nogueira et al. (2021), il a été identifié que les problèmes olfactifs et gustatifs se produisent auparavant à des intensités différentes. II n'existe aucune preuve scientifique du traitement de ces troubles.

Costa et al. (2020) ont identifié que des troubles olfactifs et/ou gustatifs peuvent survenir à des intensités variables, avec l'apparition et les symptômes antérieurs de l'infection. II n'y a aucune preuve scientifique sur le traitement de ces troubles.

Le système immunitaire humain varie en fonction de certaines caractéristiques physiques et physiologiques (Cardoso et al., 2020). L'intensité des symptômes peut survenir en raison de ces variations. Chez les femmes, les hormones produites dans les cycles menstruels, celles présentes dans la pilule contraceptive et dans le traitement de l'hormone substitutive (pour lutter contre les symptômes de la ménopause) affectent le système de défense, le rendant différent de celui des hommes (Rogero et al., 2021).

L'âge est également un facteur de différence dans l'apparition des symptômes. Contrairement à Costa et al. (2020) et Nascimento (2020), un âge plus avancé

RC: 101283

Disponible en: https://www.nucleodoconhecimento.com.br/sante/sequelles-de-lacovid-19 
semble être plus sensible aux symptômes causés par le virus. Un intestin plus âgé a généralement une absorption plus faible des nutriments et la production de cellules de défense. La différence de concept entre les auteurs peut être liée au type spécifique de symptôme (Rogero et al., 2021).

Le stress affecte également la fonction de défense immunitaire. Le système nerveux influence la production et la libération d'hormones qui peuvent servir de médiateur à cette fonction (Rogero et al., 2021). Les personnes plus jeunes, en raison de leur expérience plus courte, peuvent être plus touchées par le stress.

En ce qui concerne les traitements étudiés dans l'action contre les troubles olfactifs et gustatifs, la littérature présente l'utilisation de corticostéroïdes (action antiinflammatoire) de l'oral et de l'oral, de l'entraînement olfactif, de la vitamine $A$ et du citrate de sodium. II existe d'autres alternatives thérapeutiques, mais celles présentées ci-dessus sont plus propices à l'efficacité. Néanmoins, il existe des preuves insuffisantes ou suffisantes de l'efficacité de ces traitements (Cardoso et al., 2020; Costa et al., 2020; Felipe et al., 2021; Neta et al., 2021).

Grâce à une revue de la littérature (Brito e Silva, 2020), ils croient qu'il y a une incidence plus élevée de dommages au système nerveux dans les cas aigus du virus. Cependant, la classification des cas aigus et chroniques ne fait toujours pas l'objet d'un consensus.

Souza et al. (2021) ont conclu que les dysfonctionnements devraient être étudiés plus rigoureusement et pendant une plus longue période de temps. La COVID-19 affecte les cellules de l'épithélium olfactif, ce qui peut altérer la neurotransmission.

L'étude de Lopes et De Abreu (2021) rapporte que les séquelles causées par la maladie peuvent être de nature neurologique, au niveau cellulaire ou non. Cela peut causer des troubles, des syndromes et d'autres variables à l'avenir.

Les dysfonctionnements olfactifs et gustatifs, en général, se produisent d'une manière associée, car les sens olfactifs et gustatifs (sens chimiques, perçoivent les 
substances dans la cavité nasale et buccale) travaillent ensemble et les sensations gustatives dépendent exclusivement de l'odorat. Les sensations sensorielles se produisent en raison de l'interaction des molécules avec les récepteurs de l'odorat et du goût. Les récepteurs du goût (présents dans les régions de la langue, du palais, du pharynx, de l'épiglotte et de l'œsophage) sont responsables de l'envoi d'informations gustatives par l'influx nerveux (communication entre cellules, neurones) au système nerveux central. Dans l'odorat, l'information olfactive est également transmise au système nerveux central par des neurones qui communiquent par des courants électriques (synapses) (Neto et al., 2011; Oliveira, 2014).

Le virus COVID19 cause des lésions aux cellules neurales olfactives, car dans l'épithélium nasal, il y a une grande quantité d'enzymes qui facilitent la diffusion et la réplication de celui-ci. En réponse à l'infection, le système immunitaire " active 》 le système de défense par les cytosines (protéines), ce qu'on appelle l'effet cytopathique, qui peut compromettre le processus de neurotransmission, provoquant une perte olfactive. Cela se produit également avec le goût, où le processus inflammatoire (effet cytopathique) diminue la vie des cellules réceptrices des papilles gustatives (Cardoso et al., 2020; Felipe et al., 2021)

Les analyses scientifiques démontrent la probabilité que le virus atteigne l'épithélium nasal, et non les neurones directement. Les cellules de l'épithélium nasal conservent le soi-disant équilibre ionique du mucus, dont dépendent les neurones pour faire les synapses jusqu'au système nerveux central. Lorsque cet équilibre est altéré, l'odeur cesse de fonctionner comme il se doit, des dommages aux neurones olfactifs peuvent survenir. Pourtant, il n'y a aucune preuve si l'anosmie résulte directement du virus ou si elle est le résultat d'une réponse immunologique (Nishioka, 2020).

Au sens gustatif, les cellules réceptrices gustatives ne sont pas affectées par le virus, mais plutôt par les cellules de soutien, ce qui peut expliquer les cas de perte de goût. L'épithélium olfactif a la capacité de se régénérer, c'est-à-dire que l'anosmie est considérée comme réversible, cependant, les patients ayant une récupération lente 
de ce dysfonctionnement peuvent présenter un autre trouble, la parosmie, dans lequel les saveurs (sucrées, amères, salées, acides) sont récupérées, mais certaines odeurs et arômes deviennent désagréables (Nishioka, 2020).

Nunes et al. (2020), dans leur revue, ont constaté que l'implication du virus dans le système nerveux n'est, jusque-là, pas concluante. Comprendre les symptômes neurologiques, en particulier dans les conditions les plus graves, aide dans les interventions et les pronostics médicaux.

L'implication du virus dans le système nerveux ne semble pas concluante. Actuellement, il existe deux théories principales qui expliquent les impacts neurologiques de la COVID-19 sur l'homme, la première correspond à l'action du virus dans la dérégulation immunitaire, comme la surproduction de cytosines, et donc provoquant des symptômes neurologiques. Indiquant la possibilité que le virus se propage par le sang et les voies neuronales vers le système nerveux. La seconde suggère que l'infection du système nerveux central se produit par l'intermédiaire de neurones périphériques infectés par le virus (Felipe et al., 2021).

Brito e Silva (2020), Pimentel (2020), Santos et al. (2020) et Nogueira et al. (2021) s'accordent sur le peu de connaissances qui existent aujourd'hui et proposent la continuité des études sur les symptômes de la COVID 19. Ceci est important car grâce à des études scientifiques, il sera possible de définir les causes des mécanismes qui provoquent des changements du goût olfactif et de fournir une base pour la recherche de traitements appropriés pour les personnes touchées (Nishioka, 2020; Santos et al., 2020; Felipe et al., 2021; Lima et al., 2021; Oliveira et al., 2021).

Dans la revue de la littérature par Grendene et al. (2021), la conclusion indique que l'on ne sait pas grand-chose sur les stratégies de traitement de ce virus. C'est pourquoi des recherches cliniques sont menées pour découvrir de nouveaux traitements. La prévention aujourd'hui est toujours le meilleur moyen de faire face à l'infection. 
Les mesures de prévention contre le virus comprennent: l'hygiène des mains souvent, faite en se lavant avec du savon et de l'eau (briser la paroi lipidique du virus et le tue) et / ou en utilisant $70 \%$ d'alcool éthylique, en évitant les mains avec les yeux, le nez et la bouche, en pratiquant l'étiquetage respiratoire (soins en cas de toux ou d'éternuement), port de masques faciaux (barrière physique contre les gouttelettes pendantes), distanciation sociale d'au moins un mètre, désinfection des objets et des surfaces, isolement symptomatique, esthéstiser à l'intérieur, éviter les poignées de main et les câlins et éviter les contacts sociaux. Tous sont indiqués selon la base scientifique (Stephens et al., 2009; Baptista et Fernandes, 2020; Garcia, 2020; Soares et al., 2021; Spdm, 2021)

En ce qui concerne l'isolement social, des études indiquent son efficacité et son aide à réduire les taux d'infection, mais cela devrait être fait en association avec d'autres méditations de confinement du virus (Who, 2021). II existe également des preuves de l'aération des endroits fermés lorsqu'ils sont exposés à la lumière du soleil et de les garder ventilés, car de tels environnements contiennent les gouttelettes de vapeur d'eau provenant de la toux, des éternuements et de l'acte même de parler des individus (Opas, 2021). L'utilisation de masques est scientifiquement prouvée, on sait que cet EPI (équipement de protection individuelle) est une barrière physique, un mur, contre le virus COVID-19 (Salles et al, 2021). En outre, il est souligné que même après la vaccination, les gens ne devraient pas cesser d'utiliser / appliquer les mécanismes de prévention contre le virus (Brésil, 2021a).

Kosugi et al. (2020) ont mené une enquête en ligne et ont conclu que l'anosmie a un taux de récupération plus faible et une durée plus longue chez les patients positifs au covid-19 que chez les patients négatifs. L'hyposmie avait un taux de récupération plus élevé que l'anosmie.

Dans l'hyposmie, parce qu'il s'agit d'une perte partielle, il est possible de " entraîner » l'odeur. Les odeurs et les huiles essentielles sont utilisées deux fois par jour pendant six mois à cette fin. Perte déjà plus grave, anosmie, s'il n'y a pas de récupération instantanée, le traitement est médicamenteux et prolongé (Pereira et 
al., 2020). Cela explique probablement la différence dans les taux de récupération des deux suites.

\section{CONCLUSIONS}

Les séquelles de la COVID-19 sont diverses maladies qui compromettent les sens sensoriels (odorat et goût) à différents degrés, affectant le bien-être et la vie quotidienne de milliers de personnes.

Les femmes et les jeunes sont les plus touchés par ces dysfonctionnements. On ne sait pas exactement comment le virus provoque des symptômes neurologiques chez les patients et les moyens exacts par lesquels les dysfonctionnements se produisent. En outre, il n'existe pas de traitements spécifiques et scientifiquement prouvés aux différents dysfonctionnements, mais le plus indiqué est la pratique de l'entraînement olfactif.

Les études liées aux dysfonctionnements sont limitées, principalement, au Brésil et lorsqu'elles sont analysées comme des séquelles, c'est-à-dire étudiées après une certaine période après la guérison des patients atteints de COVID-19. La continuité de la recherche scientifique est indispensable, car seul ce processus permettra d'élucider les doutes qui existent encore et de générer des traitements appropriés pour les personnes qui ont développé la COVID-19 et qui se sont retrouvées avec certains des différents types de séquelles, y compris sensorielles.

\section{RÉFÉRENCES}

BAPTISTA, A. B.; FERNANDES, L. V. COVID-19, análise das estratégias de prevenção, cuidados e complicações sintomáticas. Revista Desafios, v. 7, p. 1-10, 2020.

BARROS, Ó. D. et al. Disgeusia: a propósito de um caso clínico. Revista Portuguesa De Medicina Geral E Familiar, v. 31, n. 4, p. 272-276, 2015. 
BRANDÃO, R. E. L. Vírus e Retrovírus: Contributo para a Evolução das Espécies. 2015. 61p. (Mestrado). Faculdade de Ciências da Saúde, Universidade Fernando Pessoa, Porto PT.

BRASIL. Coronavírus - Como é transmitido? , Brasília DF, 2021. Disponível em: < https://www.gov.br/saude/pt-br/coronavirus/como-e-transmitido >. Acesso em: 06 set 2021.

- Doenças ocasionadas por vírus respiratórios emergentes, incluindo o COVID-19. Brasília DF, 2021a. Disponível em: < https://www.unasus.gov.br/cursos/curso/46164 >. Acesso em: 06 set 2021.

BRITO, W. G. F.; SILVA, J. P. D. O. Impactos neuropatológicos do COVID-19. Brazilian Journal of Health Review, v. 3, n. 3, p. 4227-4235, 2020.

CAPES. Treinamento no uso do Portal de Periódicos. Brasilia DF, 2012. Disponível em: < https://www.fca.unesp.br/Home/Biblioteca/portal-capes.pdf >. Acesso em: 06 set 2021.

CARDOSO, A. R. Olfato como Marcador Biológico. 2018. 21p. (Mestrado). Universidade de Lisboa, Lisboa PT.

CARDOSO, M. D. C. et al. Anosmia e disgeusia no paciente com coronavírus: revisão narrativa. REAS/EJCH, v. 46, p. 1-8, 2020.

COSTA, K. V. T. D. et al. Desordens olfativas e gustativas na COVID-19: uma revisão sistemática. Braz J Otorhinolaryngol., v. 86, n. 6, p. 781-792, 2020.

ESTRELA, M. C. A. et al. Covid-19: sequelas fisiopatológicas e psicológicas nos pacientes e na equipe profissional multidisciplinar. Brazilian Journal of Development, v. 7, n. 6, p. 59138-59152, 2021. 
FELIPE, L. P. et al. Compreensão das manifestações neurológicas induzidas por infecções pelo novo coronavírus: uma revisão integrativa. REAID, v. 95, n. 36, p. e21137, 2021.

FILHO, N. D. A. O que é saúde? Rio de Janeiro RJ: Editora Fiocruz, 2011. 160p.

GARCIA, L. P. Uso de máscara facial para limitar a transmissão da COVID-19. Epidemiol. Serv. Saude, v. 29, n. 2, p. 1-4, 2020.

GOMES, D. R. D. P. et al. Avaliação do paladar de idosos e sua relação com estado nutricional e hábitos alimentares. Pan American Journal of Aging Research, v. 8, n. 1, p. 1-8, 2020.

GRENDENE, C. S.; GULO, R. B.; BETIOL, R. S. M. Coronavírus (covid-19): história, conhecimento atual e sequelas de longo prazo. Revista Corpus Hippocraticum, v. 1, n. 1, p. 1-14, 2021.

ISER, B. P. M. et al. Definição de caso suspeito da COVID-19: uma revisão narrativa dos sinais e sintomas mais frequentes entre os casos confirmados. Epidemiol. Serv. Saude, Brasília, v. 29, n. 3, p. 1-11, 2020.

KOSUGI, E. M. et al. Recuperação incompleta e tardia da perda súbita do olfato na COVID-19. Braz. j. otorhinolaryngol., v. 86, n. 4, p. 490-496, 2020.

LIMA, J. H. C. et al. Covid-19 e os danos ao aparelho olfatório causando anosmia. Revista Científica Multidisciplinar, v. 2, n. 8, p. e28665, 2021.

LOPES, D. O.; DE ABREU, F. Eletroterapia IVL no tratamento de Covid-19 e sequelas no sistema nervoso central. Brazilian Journal of Development, v. 7, n. 4, p. $42332-423402021$.

MACHADO, A. L. M.; MACHADO, M. C. M. Disgeusia: Revisão de Literatura. 2020. 56p. (Graduação). Universidade de Uberaba, Uberaba MG. 
NASCIMENTO, M. A. Alteração das funções sensoriais de olfato e paladar e seus correlatos clínicos e funcionais em indivíduos com Covid-19. 2020. 71p. (Mestrado). Universidade Federal do Rio Grande do Norte, Santa Cruz.

NETA, F. I. et al. Pathophysiology and possible treatments for olfactory-gustatory disorders inpatients affected by COVID-19. Current Research in Pharmacology and Drug Discovery, v. 2, p. 1-11, 2021.

NETO, F. X. P. et al. Anormalidades sensoriais: Olfato e paladar. Arquivos Int. Otorrinolaringol., v. 15, n. 3, p. 350-358, 2011.

NISHIOKA, S. D. A. A perda de olfato na COVID-19 já tem explicação - pelos menos em 2020. Darte. Disponível em: < https://www.unasus.gov.br/especial/covid19/markdown/335 >. Acesso em: 30 out 2021.

NOGUEIRA, J. F. et al. Distúrbios olfatórios decorrentes de infecção por SARSCoV-2: fisiopatologia, fatores de risco e possíveis intervenções. Research, Society and Development, v. 10, n. 11, p. 1-7, 2021.

NUNES, L. T. D. et al. Principais Manifestações Neurológicas decorrentes do COVID-19: uma revisão integrativa. Revista Saúde Coletiva, v. 10, n. 59, p. 42484254, 2020.

OLIVEIRA, A. A. D. et al. Análise dos principais fatores de risco preexistentes em pacientes diagnosticados com a COVID-19 no Amapá, Amazônia, Brasil. Revista Científica Multidisciplinar Núcleo do Conhecimento, v. 17, p. 56-72, 2021. Disponível em: < https://www.nucleodoconhecimento.com.br/saude/riscopreexistentes $>$.

OLIVEIRA, F. V. Aromas: contextualizando o ensino de Química através do olfato e paladar. 2014. 137 p. (Mestrado). Universidade de Santa Maria, Santa Maria RS. 
OMS. Série de documentos de informação sintéticos do escritório regional da oms para a áfrica sobre a COVID-19. Africa, p. 1-6, 2021. Disponível em: < https://apps.who.int/iris/bitstream/handle/10665/338819/WHO-AF-ARD-DAK-102021-por. pdf? sequence=1\&isAllowed=y >. Acesso em: 30 out 2021.

OPAS. Roteiro para melhorar e garantir a boa ventilação de ambientes fechados no contexto da doença causada pelo novo coronavirus, COVID-19. Washington D.C., 2021. Disponível em: < https://iris.paho.org/handle/10665.2/53938 >. Acesso em: 01 out 2021.

PEREIRA, A. C. C. M. et al. Revisão bibliográfica: Anosmia no COVID-19. Revista Cien, v. 15, n. 2, p. 96-, 2020.

PIMENTEL, B. N. As disfunções olfativas e gustativas como apresentação clínica da COVID-19. Research, Society and Development, v. 9, n. 8, p. e64985072, 2020.

ROGERO, M.; NATACCI, L.; AMANCIO, O. Nutrição \& Imunidade. São Paulo SP: SBAN, 2021. 27p.

SANTO, D. M. N. D. E. et al. Desafios do enfermeiro do Centro Cirúrgico frente à pandemia da COVID-19 e transição de uma sala cirúrgica em unidade de terapia semi-intensiva. REAS, v. 13, n. 6, p. 1-6, 2021.

SANTOS, I. H. A. et al. Disfunções olfativas e gustativas na COVID-19. Research, Society and Development, v. 9, n. 12, p. e42591211363, 2020.

SESAPR. Reabilitação do paciente pós tratamento de infecção por SARS- CoV-2. Curitiba PR, 2020. Disponível em: < https://www.saude.pr.gov.br/sites/default/arquivos_restritos/files/documento/202012/Nota\%20orientativa\%20n\%C2\%BA\%2053\%20reabilita\%C3\%A7\%C3\%A3o.pdf >. Acesso em: 09 set 2021. 
SILVA, A. W. C. et al. Caracterização clínica e epidemiologia de 1560 casos de COVID-19 em Macapá/AP, extremo norte do Brasil. Research, Society and Development, $\quad$ v. $9, \quad$ n. $8, \quad$ p. 1-21, 2020a. Disponível em: < https://rsdjournal.org/index.php/rsd/article/view/5499/4641 >.

SILVA, A. W. C. et al. Perfil epidemiológico e determinante social do COVID-19 em Macapá, Amapá, Amazônia, Brasil. Revista Científica Multidisciplinar Núcleo do Conhecimento, v. 4, n. 4, p. 05-27, 2020. Disponível em: < https://www.nucleodoconhecimento.com.br/saude/covid-19-em-macapa >.

SOARES, K. H. D. et al. Medidas de prevenção e controle da covid-19: revisão integrativa. Revista Eletrônica Acervo Saúde, v. 13, n. 3, p. 1-11, 2021. Disponível em: < https://doi.org/10.25248/reas.e6071.2021 >.

SOUZA, F. D. S. et al. "Anarquia do paladar" e anosmia pós-COVID-19: Seriam danos permanentes? Relato de casos. Revista de Ciências Biológicas e da Saúde, v. 4, n. 1, p. 1-6, 2021.

SOUZA, K. O. et al. Covid-19 e o cenário atual da Cidade de Castanhal PA, Brasil. Research, Society And Development, v. 9, n. 8, p. 1-17, 2020. Disponível em: < https://rsdjournal.org/index.php/rsd/article/view/5717/4998 >.

SPDM. HMB explica por que a higienização das mãos auxilia na prevenção da Covid-19. São Paulo SP, 2021. Disponível em: < https://www.spdm.org.br/saude/noticias/item/3390-hmb-explica-por-que-ahigienizacao-das-maos-auxilia-na-prevencao-da-covid-19 >. Acesso em: 04 out 2021.

STEPHENS, P. R. S. et al. Virologia. In: MOLINARO, E. M.;CAPUTO, L. F. G., et al (Ed.). Conceitos e métodos para a formação de profissionais em laboratórios de saúde. Rio de Janeiro RJ: EPSJV, v.4, 2009. p.125 - 220. 
THEY, N. H. Você sabe o que é um vírus? Microbiologando Porto Alegre RS, 2020. Disponível em: < https://www.ufrgs.br/microbiologando/voce-sabe-o-que-eum-virus/ >. Acesso em: 06 set 2021.

UZUN, A. C. D. S. B. Sequelas da Covid-19 vão além da fadiga, ressalta especialista. Campo Grande MS, 2020. Disponível em: < http://www.ms.gov.br/sequelas-da-covid-19-vao-alem-da-fadiga-ressalta-especialista/ >. Acesso em: 06 set 2021.

VANA, L. P. M.; SCHIOZER, W. Sequelas de queimaduras: nova classificação. Rev Bras Queimaduras, v. 12, n. 3, p. 192, 2013.

Soumis: Novembre, 2021.

Approbation: Novembre 2021. 